\title{
Agricultura, cultura del oasis y megaminería en Mendoza. Debates y disputas
}

Agriculture, oasis' culture and mega-mining in Mendoza. Debates and disputes

Lucrecia Wagner

Grupo de Historia Ambiental, Instituto Argentino de Nivologia, Glaciologia y Ciencias Ambientales, CONICET, Argentina lucrewagner@gmail.com

\section{ReSUMEN:}

Mendoza es una de las provincias argentinas donde el rechazo a la megaminería ha sido más exitoso. Este trabajo tiene como objetivo analizar la cultura mendocina del oasis como uno de los fundamentos de la oposición a esta actividad. Basándonos en fuentes documentales, producidas principalmente por el Estado provincial, y en un trabajo etnográfico que recupera testimonios de quienes se oponen a la megaminería, concluimos que el rechazo a esta actividad tiene un arraigo en ciertas características de la historia provincial, que combinadas con nuevas demandas socioambientales, han dado lugar a una de las resistencias a la megaminería más trascendentes de la Argentina.

Palabras Clave: Mendoza, Oasis, Argentina, Conflicto ambiental, Minería.

\section{ABStract:}

Mendoza is one of the argentinean provinces where the rejection of the mega mining has been most successful. This work aims to investigate the oasis culture, as one of the basis of opposition to this activity. Based on documentary sources, produced mainly by the provincial government, and an ethnographic work that includes testimonies of the protagonists of mega mining rejection, we conclude that the rejection of this activity is rooted in certain provincial history characteristics, which combined with new socialenvironmental demands, have given rise to one of the most transcendent resistance to mega mining in Argentina.

KEYWORDS: Mendoza, Oasis, Argentina, Environmental conflict, Mining.

\section{INTRODUCCIÓN}

En los últimos 15 años, el auge de los intentos por explorar y explotar proyectos mineros a gran escala en la provincia de Mendoza -Argentina- despertó diferentes voces y posicionamientos. La primera resistencia a un proyecto minero metalífero se generó desde el año 2003 en el departamento de San Carlos (oasis centro de la provincia), que junto a los departamentos de Tunuyán y Tupungato conforman el Valle de Uco. Las preocupaciones centrales en relación a los proyectos mineros a gran escala han sido el consumo de agua, y la potencial contaminación que esta actividad pudiera provocar sobre los recursos hídricos, debido principalmente a las sustancias utilizadas (cianuro, ácido sulfúrico, entre otras). En el año 2005, la protesta contra la minería metalífera en Valle de Uco se manifestó en el espacio público, especialmente a partir de la aparición de los vecinos autoconvocados en algunos actos oficiales y la realización de manifestaciones masivas sobre la Ruta Nacional N. ${ }^{\circ}$ 40. Posteriormente, con la conformación de otros grupos de resistencia a la megaminería en la provincia, a la preocupación por el uso y contaminación de las fuentes de agua se fueron sumando otros cuestionamientos, como la falta de información brindada por las empresas sobre los impactos del proyecto, el modelo de "desarrollo" que esta actividad representa, y sus consecuencias sociales, entre otros. Este trabajo tiene como objetivo principal analizar la cultura mendocina del oasis como uno de los fundamentos de la oposición a la megaminería, tanto desde los colectivos sociales que se conformaron para resistir la actividad, como también desde los gobiernos provinciales que defendieron la constitucionalidad de la legislación sancionada en el marco del conflicto. Como objetivo secundario, buscamos dar cuenta de las discusiones sobre los modelos de desarrollo que abrió la potencial llegada de la 
megaminería, cómo se ambientalizaron estos debates, y cómo el manejo del agua ocupa un rol central en estas disputas en el oeste argentino.

Megaminería -o proyectos mineros a gran escala- define a un tipo específico de actividad minera, que ingresa al país en la década de los 90, con el fin de explotar yacimientos minerales de baja ley (menor cantidad de mineral por roca extraída). El prefijo mega no se restringe solo a la magnitud del proyecto minero, incluye también otras características de este tipo de minería, tales como: explotación a cielo abierto; generación de pasivos ambientales importantes (open pit o rajo abierto, escombreras, diques de cola, etc.); extracción acelerada de recursos naturales no renovables; uso de sustancias potencialmente contaminantes; gran consumo energético; utilización de importantes volúmenes de agua; generación de drenaje ácido de mina (DAM) y potenciación del drenaje ácido de roca (DAR); procesos que implican la liberación de metales pesados; apertura de caminos y aumento de tráfico, entre otros impactos. Es decir, la megaminería posee características que comparte con otros tipos de minería, a las que se suman características propias, muchas de las cuales responden a la envergadura de la ocupación espacial que estos proyectos requieren. A estos impactos ambientales se han sumado en el devenir de la conflictividad los impactos específicamente sociales, como la división social generada en ciertas comunidades (Wagner, 2012) y las estrategias desarrolladas por empresas, gobiernos y otros actores afines, para imponer el imaginario y el discurso pro-minero (Antonelli, 2014; Godfrid, 2016).

Lo que se contiende es la gran minería metalífera orientada a los mercados externos, basada en el alto precio internacional del oro, las buenas condiciones geológicas de los yacimientos, la legislación benevolente, el acuerdo político provincial y nacional y la compra de voluntades con todo tipo de dádivas a los vecinos para lograr la denominada Licencia Social a manos de los consorcios. Sin ésta, el Banco Mundial no otorgaría los créditos que muchas de las empresas solicitan para realizar los emprendimientos (Bueno, 2014, p. 108).

Como se mencionó previamente, en Mendoza, las primeras movilizaciones en rechazo a la actividad megaminera se llevaron adelante en el oasis centro, zona denominada Valle de Uco. Las movilizaciones posteriores fueron en el oasis sur, más precisamente en el departamento de General Alvear y en algunas localidades del departamento de San Rafael, donde también se habían producido acciones de oposición a la reapertura de la mina de uranio Sierra Pintada.

Con el agua como eje central de la oposición a los megaproyectos mineros, el debate sobre la coexistencia del perfil agrícola de Mendoza con la minería metalífera fue ganando visibilidad. En este trabajo indagaremos en los aspectos agrícolas y ambientales que, por configurar un imaginario ambiental mendocino y una denominada "cultura del oasis" impregnaron no sólo las acciones colectivas de rechazo a la megaminería, sino también las respuestas que desde el Estado provincial se dieron para defender la constitucionalidad de leyes que restringieron y/o prohibieron la actividad minera. Los sectores que rechazan la actividad minera a gran escala no son exclusivamente productores agrícolas. Pero incluso aquellos que no lo son, comparten las miradas sobre una provincia históricamente dedicada a la agricultura, y más incipientemente al turismo, actividades que en las últimas décadas se pone en riesgo ante la llegada de este tipo de minería.

A continuación desarrollaremos en primer lugar los antecedentes en los que se inscribe este trabajo, y las formulaciones teóricas que nos permiten dar cuenta de los procesos sociales que acontecieron en el territorio mendocino, explicando principalmente las nociones de imaginario ambiental mendocino y cultura del oasis. Posteriormente, contextualizaremos el debate en los conflictos que se desarrollaron en Mendoza por los proyectos de megaminería que intentaron llevarse adelante, con el fin de fundamentar cómo esta cultura del oasis ha sustentado tanto la resistencia social ante los proyectos megamineros, como la postura del gobierno provincial al defender leyes que limitan y prohíben esta actividad en territorio mendocino. A continuación, abordaremos otras contribuciones que aportan a la discusión de la relación entre los conflictos ambientales por megaminería y los modelos de desarrollo en disputa en Mendoza. Finalmente, desarrollaremos las reflexiones a las que hemos arribado. 


\section{ANTECEDENTES Y FORMULACIONES TEÓRICAS}

Los conflictos relacionados a la megaminería están presentes en diferentes lugares del mundo, y América Latina es una de las regiones que más conflictos de este tipo posee ${ }^{1}$.

Estos procesos han sido objeto de diversas publicaciones colectivas sobre sus características en la región (Delgado Ramos, 2010; Alimonda, 2011; Göebel y Ulloa, 2014; Deonandan and Dougherty, 2016; Bottaro y Sola Álvarez, 2018). También en Argentina, el tema de los conflictos por megaminería ha sido eje de obras colectivas (Svampa y Antonelli, 2009; Machado et al., 2011) y ha sido tema de numerosos dossiers de revistas, como Theomai (Galafassi, 2013), Entramados y Perspectivas (Hadad, 2013), RevIIse (Giraud y Wagner, 2017), y de tesis de grado y posgrado (Schiaffini, 2003; Claps y Colao, 2005; Wagner, 2010; Sola Alvarez, 2012; Machado Araoz, 2012; Walter, 2014), entre otras.

Este trabajo surgió a partir de una investigación sobre el conflicto socioambiental acontecido en Mendoza por la potencial instalación de proyectos megamineros (Wagner, 2010; Wagner, 2014). Si bien hay un conflicto ambiental cuando un conjunto de actores sociales plantea demandas en el terreno político en torno a la apropiación social de bienes de la naturaleza, o donde se pone en juego un argumento ambiental, es importante entender que esos reclamos pueden estar mediados por otras demandas de naturaleza social, económica y cultural. "La nominación ambiental se establece cuando en la dinámica contenciosa los actores utilizan argumentos ambientales, aun si estos no son los prevalecientes” (Merlinsky, 2013, p. 40).

Como destaca José Sergio Leite Lopes (2006), puede observarse un proceso de “ambientalización” de diferentes espacios, temáticas y conflictos. Esta denominación designa tanto al proceso de adopción de un discurso ambiental genérico por parte de los diferentes grupos sociales, como también la incorporación concreta de justificativos ambientales para legitimar prácticas institucionales, políticas, científicas, etc. Su pertinencia teórica, entonces, gana fuerza particular en la posibilidad de caracterizar procesos de ambientalización específicos a determinados lugares, contextos y momentos históricos. Disputas de legitimidad se instauran en la búsqueda por caracterizar las diferentes prácticas como ambientalmente benignas o dañosas. En esas disputas, en las que diferentes actores sociales ambientalizan sus discursos, acciones colectivas son esbozadas en la constitución de conflictos sociales incidentes sobre esos nuevos objetos, sea cuestionando los padrones técnicos de apropiación del territorio y sus recursos, sea contestando a la distribución de poder sobre ellos (Acselrad, 2010). Así, en los últimos años asistimos cada vez más, en palabras de Maria Carman (2011), a la emergencia de una argumentación ambiental cuyo papel es central en la conformación de la legitimidad de determinadas políticas públicas.

En este trabajo argumentamos que esta ambientalización del conflicto en torno a la megaminería en Mendoza constituyó un éxito para los colectivos sociales que organizaron la resistencia a esta actividad. Y planteamos también que las argumentaciones de la oposición a la megaminería estuvieron sustentadas en el imaginario ambiental mendocino, lo cual colaboró en dotar de legitimidad a las demandas socioambientales de las asambleas por el agua mendocinas.

La noción de imaginario ambiental mendocino fue trabajada junto a otros colegas en un artículo titulado "Imaginario ambiental mendocino: sus efectos en las políticas estatales y la producción científica" (Escolar, Martín, Rojas, Saldi y Wagner, 2012), y hace referencia al proceso iniciado ya desde mediados de siglo XIX, en el que van cobrando fuerza un conjunto de imágenes y argumentos que, para explicar la excepcionalidad cuyana y en particular mendocina y sanjuanina, asociaron una serie de condiciones naturales a características sociopolíticas de su población. Mostramos cómo estas nociones llegaron a constituirse como una doxa que influyó en la producción científica local, en políticas estatales y prácticas de gobierno hasta la actualidad. Particularmente, el conflicto en torno a la megaminería, por ser el de mayor y más rápida instalación pública de la última década, fue utilizado para analizar las reactualizaciones y la productividad política del imaginario ambiental fundacional. 
Entre las implicancias en políticas estatales se destaca la sustentación de la legislación existente en materia ambiental desde una "cultura ambiental del oasis", postulado que también va a formar parte de las retóricas presentes en los conflictos que tengan como eje de la disputa el recurso estratégico de Mendoza: el agua (Escolar, Martín, Rojas, Saldi y Wagner, 2012).

La preservación del ambiente como objetivo político de la Provincia de Mendoza no es un fenómeno coyuntural, un hecho accidental, sino que es la consecuencia de un largo proceso cultural que encuentra en la historia provincial sus verdaderas bases. La cultura mendocina es la cultura ambiental del oasis (Abraham, Rodríguez Salas, 1993, p. 14).

El imaginario ambiental actuó así como un dispositivo que operó tanto como origen de muchas explicaciones científicas y políticas estatales, al tiempo que él mismo se reconfiguraba a partir de éstas. Un imaginario que no ha estado exento de tensiones y contradicciones, sino todo lo contrario. En el artículo ya destacado (Escolar, Martín, Rojas, Saldi y Wagner, 2012) nos preguntábamos precisamente cómo avanzar en la discusión sobre alternativas para estas zonas, que no tuvieran como impronta la imposición de un modelo propuesto desde una toma de decisiones que prioriza el oasis, o cómo establecer una retórica convincente en el debate sobre proyectos extractivos -como la minería a gran escala, o la actividad petrolera- que no estuviese amparada en una legitimación de la apropiación desigual del agua existente desde antes del siglo XX. Sólo recientemente, en parte alimentado por la ambientalización del derecho de aguas, pero fundamentalmente por la explosión de diversos conflictos ambientales, muchos de ellos protagonizados por movimientos sociales y asambleas, están emergiendo otras voces que, aunque no siempre antagónicas con este imaginario, plantean nuevos interrogantes e ideas que tensionan y recrean este imaginario ambiental (Escolar, Martín, Rojas, Saldi y Wagner, 2012).

Otra noción que sustenta este trabajo es la de "cultura del oasis". Con este concepto buscamos definir un conjunto de ideas, memorias y conocimientos que han permitido construir una identidad mendocina ligada a la conformación de los oasis, los cuales constituyen una herencia que enorgullece a la provincia, ya que los actuales pobladores de Mendoza, especialmente quienes habitan en zonas agrícolas, destacan que fueron sus antecesores (padres, abuelos, bisabuelos) quienes lograron llevar a cabo y mantener oasis agrícolas en un medio árido. Esta cultura del oasis, que forma parte de la historia de sus habitantes, está fomentada también desde las instituciones gubernamentales provinciales, principalmente aquellas vinculadas a temas hídricos y ambientales.

Esta noción aparece también en la argumentación sobre la defensa del agua, por parte de los colectivos que resisten a la megaminería. Por ejemplo, la Multisectorial del Sur, una de las organizaciones que se ha opuesto a la minería del uranio en San Rafael, al presentar un recurso de amparo solicitando la protección de los glaciares destacó: "Los glaciares brindan importantes servicios ambientales, por cuanto han permitido la cultura del oasis en todo el oeste del país, la agricultura, la vitivinicultura, el turismo, la generación de energía eléctrica, etc." (Multisectorial del Sur, 2009) ${ }^{2}$.

Investigadores mendocinos también hacen uso de este concepto. "La cultura mendocina es una cultura del oasis, forjada en la necesidad imperiosa de su pueblo de conocer el medio para poder administrarlo, preservarlo y desarrollarlo", destacan Elena Abraham y Aldo Rodríguez Salas (2003). Estos autores mencionan algunas características ambientales, económicas y sociales de Mendoza que son importantes para contextualizar nuestro tema:

Los $220 \mathrm{~mm}$ anuales de lluvia, que precipitan en pocos meses del año, exigen una economía del recurso hídrico que se obtiene sólo de la nieve acumulada en la cordillera de Los Andes. A partir de sus principales ríos, se han formado los oasis irrigados (Norte, Central y Sur) en los que se concentra la vida productiva, social y política. En el Oasis Norte se asienta el 70\% de la población. Cuenta aproximadamente con 360.000 ha sistematizadas para riego de uso agrícola, superficie que no llega al $3 \%$ de la superficie total de la provincia $(150.839 \mathrm{~km} 2)$. No debe sorprender entonces que desde el siglo pasado haya comenzado a gestarse una sostenida política legal dirigida a la preservación y correcta administración de los recursos naturales, en especial del recurso hídrico. La Ley General de Aguas del año 1884 fue la primera en su género en el país y su vigencia actual demuestra 
el adelanto institucional que significó para la administración del recurso hídrico y el desarrollo de la economía provincial (Abraham y Rodríguez Salas, 2003, p. 2)

\section{Decisiones metodológicas}

Esta investigación fue desarrollada con base en metodología cualitativa, basada en el análisis de fuentes documentales, tanto gubernamentales, como empresariales y de las organizaciones sociales que se conformaron para resistir a los proyectos megamineros, y la realización de entrevistas en profundidad a diferentes actores del conflicto. En cuanto a las fuentes gubernamentales, se consultaron expedientes de evaluación ambiental de proyectos mineros, versiones taquigráficas de debates legislativos provinciales (de aquellos que derivaron en la sanción de legislación relacionada a la actividad minera), leyes provinciales, y presentaciones legales de la provincia (a través de su Asesoría de Gobierno) en defensa de ciertas leyes provinciales (Ley 7422 y Ley 7722). Se consultaron también páginas webs de las empresas y cámaras empresarias mineras, sus informes de impacto ambiental y comunicados públicos. También se analizaron comunicados de las asambleas de vecinos autoconvocados y otros colectivos, y la información por ellos reunida sobre presentaciones y pedidos de informe a diferentes autoridades y entidades del gobierno municipal y provincial. Estas fuentes documentales fueron complementadas con entrevistas en profundidad. Se entrevistaron alrededor de diez integrantes de las organizaciones con más trayectoria desde el inicio del conflicto, localizadas en diferentes regiones de la provincia, y que organizaron las acciones más emblemáticas contra la megaminería en la provincia, entre el año 2003 y el $2011^{3}$. En el resto de las organizaciones se entrevistaron entre tres y cinco integrantes. Los integrantes entrevistados fueron elegidos en función de su trayectoria en la organización, su participación en hechos claves, y se buscó además priorizar la heterogeneidad de género y edades, a fin de contar con diferentes apreciaciones de los acontecimientos y garantizar la heterogeneidad de la muestra. Es importante destacar que la mayor parte de las acciones aquí detalladas fueron organizadas en conjunto por diferentes colectivos, y apoyadas por otros movimientos sociales que varían según la región donde se llevó a cabo la acción. En este sentido, es importante destacar que las entrevistas estuvieron dirigidas a miembros de las organizaciones (asambleas por el agua y otros colectivos) nucleadas en la Asamblea Mendocina Por el Agua Pura (AMPAP). También se entrevistaron representantes de las áreas de Ambiente y Minería, y de la Asesoría de gobierno provinciales, y en algunos casos municipales. Complementariamente, se concurrió a diversos eventos gubernamentales, empresariales, universitarios y de las organizaciones en resistencia, en relación al conflicto socioambiental despertado por la llegada de proyectos megamineros.

\section{MENDOZA, TIERRA DE AGUA Y OASIS}

La ampliación de la Reserva Laguna del Diamante asegurará la calidad del recurso hídrico, tanto superficial como subterráneo, en toda la zona alta de la cuenca del río Tunuyán Superior, así como también por supuesto hacia aguas abajo, donde están los usos agrícolas del recurso vital, agua (...) indudablemente traerá un gran beneficio económico para la región del Valle de Uco. Una parte sustancial de la producción agrícola en países de la región depende de las cuencas hidrográficas altoandinas (Asesoría de Gobierno, 2005, pp. 36-39).

El párrafo previo es un extracto de la defensa que llevó adelante el gobierno de la provincia de Mendoza, ante la demanda iniciada por una empresa minera, Anglo American, contra la ley No 7422, de ampliación de la reserva Laguna del Diamante, en el año 2005. Esta ley de ampliación se había sancionado en el marco de las primeras movilizaciones contra un proyecto minero metalífero en las cercanías de la reserva Laguna del Diamante, en San Carlos (Valle de Uco). Debido a la ampliación del área de reserva, los derechos mineros de esta empresa, y de otras, quedaron dentro de la misma, por lo cual quedaba prohibida su actividad de exploración ${ }^{4}$. La empresa minera consideraba que esta ley era inconstitucional; argumentaba falta de motivo 
o razonabilidad, derechos adquiridos, derechos de propiedad, imprecisión de límites de la reserva, entre otras razones. Ante ello, el gobierno de Mendoza defendió la constitucionalidad de la Ley; respondió a los cuestionamientos de la empresa desde el derecho ambiental, la legislación provincial sobre áreas protegidas, e hizo referencia al contexto social en el que se generaban las declaratorias de áreas naturales protegidas. Por ello, en este trabajo se utiliza la noción imaginario ambiental (Escolar, Martín, Rojas, Saldi y Wagner, 2012), y no exclusivamente agrícola ${ }^{5}$, porque la posibilidad de desarrollar megaproyectos de minería, además de percibirse como una actividad que pondría en riesgo el perfil productivo de Mendoza, también colisionó con la creación, existencia y/o ampliación de áreas naturales protegidas, y con algunos principios del derecho ambiental, en los cuales Mendoza ha basado su legislación en esta materia.

A fin de contextualizar estos debates, es importante relatar que San Carlos es un departamento que se encuentra en el centro-oeste de la provincia, a $100 \mathrm{~km}$ aproximadamente de la ciudad capital y, junto a los departamentos Tunuyán y Tupungato, conforma la zona denominada Valle de Uco. Como ya fue destacado, actividades de exploración en las cercanías de un área natural protegida, la Laguna del Diamante, generaron el alerta de algunos habitantes de este departamento, quienes comenzaron a reunirse para informarse sobre el tema, y decidieron rechazar la actividad minera en las cercanías de fuentes de agua ${ }^{6}$. Luego de numerosas actividades de difusión al resto de la población -a las que también se sumaron pobladores de Tunuyán y Tupungato, y que fueron realizadas entre los años 2003 y 2004-, en 2005 las manifestaciones públicas para rechazar a los proyectos mineros reunieron a más de 4.000 personas en los alrededores de la Terminal de buses de Eugenio Bustos (San Carlos), por donde pasa la Ruta Nacional N $40^{7}$.

Ante estos hechos, la posibilidad de frenar el proyecto minero que inauguró la conflictividad socioambiental por megaminería en Mendoza estuvo dada por la ley de ampliación de la reserva natural Laguna del Diamante, que, como ya fue destacado, fue demandada de inconstitucionalidad por el sector minero. 
Figura 1: Sitios relevantes para el conflicto por megaminería en Mendoza

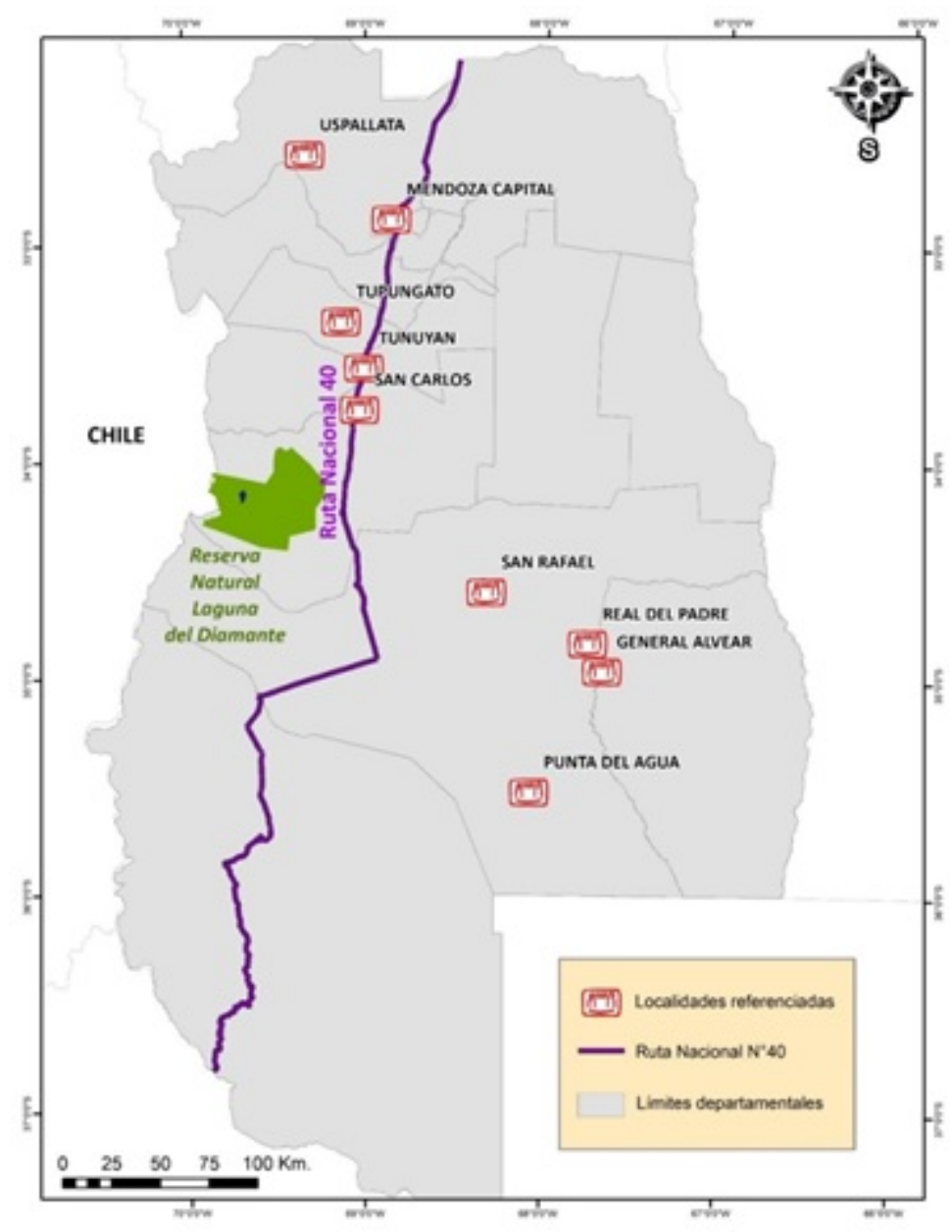

Fuente: Elaborado por Facundo Rojas (2018)

La defensa de la constitucionalidad de la ley por parte del Gobierno provincial, incluye argumentos que coinciden con la preocupación de los pobladores del Valle de Uco sobre la posibilidad de compatibilizar las actividades existentes con la llegada de la minería a gran escala:

La zona del Valle de Uco es un área agrícola con excelentes productos derivados de la agricultura, tanto para consumo interno como para exportación, irrigados con un agua que hoy en día muestra una excelente aptitud para agricultura de regadío. La minería a cielo abierto, de metales preciosos como el oro, trae aparejada una serie de impactos negativos sobre los recursos del ambiente en virtud de la utilización, entre otros, de cianuro, una gran cantidad de residuos de material rocoso también queda disperso en el área de explotación, así como también el potencial peligro de afectación con drenajes ácidos del agua subterránea subyacente (Asesoría de Gobierno, 2005, p. 37)

Sumado a ello, el gobierno provincial destacó en su defensa que resultaba "paradigmático" el consenso existente en la comunidad del departamento donde se localiza el área protegida ampliada, San Carlos, a favor de esta ampliación. Resalta además que fue esta comunidad la que mediante audiencias, plenarios, publicaciones periodísticas y movilizaciones solicitó a la Legislatura provincial la sanción de la ley, en el convencimiento de que era la herramienta legal más segura e integral para la preservación de los recursos naturales de la zona, en especial el recurso hídrico. En este sentido, la Asesoría de Gobierno, encargada de la defensa de la ley, argumentó que este departamento, al igual que el resto de la región conocida como el Valle de Uco, basa su economía principalmente en la actividad agrícola, "actividad que históricamente fue el sustento de la población local constituyendo entonces la esencia cultural de ese pueblo” (Asesoría de Gobierno, 2005, pp. 60-61). 
El diario provincial Los Andes, uno de los principales medios de prensa escrita de la provincia, destaca la movilización realizada por los vecinos de San Carlos para pedir que no se realizara minería metalífera en la zona de la Laguna del Diamante:

Hace una semana se reunieron 4 mil personas (el $15 \%$ de su población), una convocatoria voluntaria que, hoy por hoy, es envidia de cualquier agrupación política. La movilización fue para reclamar al Gobierno que no permita la minería en las cercanías de la reserva Laguna del Diamante porque, aseguran, puede contaminar ${ }^{8}$.

La potencial contaminación de la Laguna del Diamante aparece como el primer motivo del rechazo a la megaminería en San Carlos, al que luego, como ya se destacó, se fueron sumando otros argumentos: "Del saqueo no teníamos ni idea, la preocupación era que nos tocaran la reserva de la Laguna del Diamante y que nos contaminaran el agua con el método" (vecino de San Carlos, citado en Wagner, 2014, pp. 286) ${ }^{9}$.

Además, la Asesoría comparó esta actividad con la potencial actividad minera, y fundamentó así el accionar de los pobladores de San Carlos y de los legisladores:

Cabe acotarse que San Carlos es el departamento con menor índice de desocupación de toda la provincia. No resulta
descabellado entonces que una población que ha sabido sostenerse a través del tiempo haciendo un uso adecuado y racional de
sus recursos naturales renovables quiera protegerlos, y con ellos su forma de vida, frente a una aventura altamente impactante
como lo es la actividad minera, con dudosos beneficios en el corto plazo para la población local y serios perjuicios en el
largo plazo para la humanidad toda. Tampoco resulta "caprichoso" y mucho menos "inconstitucional" que el legislador
haya receptado el reclamo de una comunidad que demanda la protección de sus derechos, sancionando la Ley en cuestión
(Asesoría de Gobierno, 2005, p. 61).

Este imaginario que destaca la importancia del agua, el oasis y la agricultura como ejes de la preservación, enraizado en la historia provincial, se manifiesta también en la legislación ambiental de Mendoza, sancionada a inicios de la década de 1990, y base de sus políticas ambientales, ancladas en la importancia del agua para la organización del territorio. Precisamente, la defensa del gobierno ante las acciones de inconstitucionalidad del sector minero se sustenta en esta legislación.

Nuestra investigación, iniciada en 2007 y llevada adelante por más de 10 años en la provincia de Mendoza pero atenta al contexto nacional de la conflictividad socioambiental, nos ha permitido constatar que los argumentos esgrimidos por el gobierno mendocino para responder al sector minero que pretendía instalarse en la provincia coinciden en gran parte con los de los referentes de las movilizaciones socioambientales en Argentina, que acompañaron en algunos momentos las resistencias a la megaminería en Mendoza. En este sentido, Javier Rodríguez Pardo - periodista y ambientalista, que integró las movilizaciones contra el repositorio nuclear en Gastre, a mediados de la década del 80- afirmaba en su visita a Mendoza, en el año 2005: "Dije entonces, y no me equivoqué, que Mendoza iba a ser un hueso duro de roer, impidiéndole a las mineras hacer base, porque enseguida se nota la importancia que el agua tiene en una población que hizo de un desierto un vergel” (Rodríguez Pardo, 2009, pp. 225-226) ${ }^{10}$.

La evocación del trabajo realizado para "vencer al desierto" está presente en muchos de los testimonios recabados durante las entrevistas realizadas a los integrantes de las asambleas y grupos de vecinos autoconvocados que se organizaron para rechazar la megaminería, quienes a fines del año 2006 se nuclearon en AMPAP (Asamblea Mendocina por el Agua Pura) ${ }^{11}$. En estos testimonios aparecen repetidamente dos cuestiones: por un lado, el orgullo y la necesidad de preservar el trabajo de sus antepasados y, por otro, la denuncia de la invisibilización e injusticia con ciertos sectores de la población mendocina, que sufren históricamente problemas de acceso al recurso hídrico. Estos aspectos también son destacados por investigadores mendocinos, entre ellos Rodolfo Richard-Jorba y Elma Montaña: "por estar situadas [Mendoza y San Juan] en la diagonal árida sudamericana, han estructurado sus economías en oasis agrícolas construidos y organizados por ciudades, en los que se concentra la población y se desarrollan los aspectos fundamentales de su vida social y económica" (Richad-Jorba, Pérez Romagnoli, Barrio y Sanjurjo, 2006, p. 21). 
Esa identidad de los mendocinos es el fruto de una construcción histórica que combina diversos factores geográficos, demográficos, culturales y políticos. Entre ellos destaca, como un modo de vida más o menos compartido, la organización de la sociedad local en torno a una causa común: "vencer al desierto". Los mendocinos están orgullosos de la manera en la que han sabido "domar" ese medio hostil y moldearlo de acuerdo a sus necesidades (Montaña, Torres, Abraham, Torres y Pastor, 2005, p. 5)

Esta identidad se refleja en los testimonios que recabamos de los miembros de las asambleas por el agua conformadas en aquellos departamentos con mayor tradición agrícola, especialmente en Valle de Uco y General Alvear, como así también en San Rafael. En este sentido, una vecina de Punta del Agua, localidad en la que se inicia el conflicto que va a dar lugar a las concurridas movilizaciones contra la actividad minera metalífera en General Alvear, destaca:

\begin{abstract}
No podemos hablar de trabajar la minería como única salida laboral para la provincia, y menos con lo que nos va a pasar en el futuro con el tema de las reservas de agua, el tema de conservar glaciares, la importancia de nuestra provincia, de que nuestros antepasados lograron hacer un oasis de este desierto, usando el agua de los deshielos de los glaciares, y no podemos, con la minería, darnos el lujo de destruir los glaciares para destruir todo lo que se ha logrado a través de los años (vecina de Punta del Agua, citada en Wagner, 2014, pp. 167-168).
\end{abstract}

Otro testimonio de un integrante de las asambleas mendocinas da cuenta de la misma percepción en torno al pasado y presente mendocinos: "Es una realidad de nuestra provincia, somos la provincia de las acequias, de los canales, somos una provincia que se superó haciendo un uso racional del agua” (vecino de General Alvear, citado en Wagner, 2014, p. 168).

Las movilizaciones en General Alvear fueron acompañadas por pobladores de algunos distritos del departamento de San Rafael, como Real del Padre. En los testimonios de estos pobladores también se refleja la defensa del perfil productivo local: "El pueblo es agrodependiente, es la capital del damasco, también hay viñedos, ciruelas, peras (...) hay dos exportadores de frutas al exterior, uno de vinos y luego chiquitos al interior del país" (vecina de Real del Padre, citado en Wagner, 2014, p. 185). Una productora de este distrito relata lo sucedido en los cortes de ruta que llevaron adelante en 2007, en los que se solicitaba la sanción de una ley que prohibiera la actividad minera metalífera en la provincia ${ }^{12}$ : "las panaderías donaban masa y a la tarde se tomaba mate con torta fritas. Con días de lluvia, se armaron carpitas y nos quedábamos ahí” (vecina de Real del Padre, citado en Wagner, 2014, p. 186). A continuación, esta vecina de Real del Padre afirma:

...todo por defender lo nuestro, lo que tanto han hecho nuestros padres, nuestros abuelos, esto era un desierto y esto se hizo a fuerza, no de tractores, a lomo de burro y caballos (...) así hicieron todo nuestros abuelos (...) principalmente el agua, si se contaminan los ríos nosotros desaparecemos (...) (citado en Wagner, 2014, p. 186).

Desde las autoridades ambientales del municipio de General Alvear, también se planteaba el problema existente entre la agricultura y la competencia con la minería metalífera por el recurso hídrico:

\begin{abstract}
No es toda la minería, el problema es la minería metalífera y en la escala que se quiere hacer (...), la nuestra no es una postura ambientalista, es una postura a favor del desarrollo sustentable. La minería en este rubro viene a competir con las actividades que ya existen, y con la cultura de producción de cada lugar donde ellas van, y al afectar los recursos básicos, básicamente acá, aunque no contaminaran, hagamos el supuesto de que nunca contaminarían, la competencia por el recurso hídrico es muy importante, después te va a faltar agua para la agricultura, y en un desierto como es Mendoza, el recurso hídrico es muy limitado. Entonces, aparte de la contaminación en sí, hay otros factores para tener en cuenta (Funcionario del Área Ambiental del municipio de General Alvear, citado en Wagner, 2014, p. 186)
\end{abstract}

En el año 2007, los conflictos por minería metalífera en Mendoza alcanzaron su momento más álgido. A fines del año 2006, las protestas provenientes de Valle de Uco, General Alvear y San Rafael, a las que se sumaron organizaciones y particulares del Gran Mendoza, se concentraron en la Legislatura provincial para solicitar una ley que impidiera la minería metalífera en la provincia. Se sancionó así una ley ${ }^{13}$ que frenaba la actividad hasta tanto Mendoza contara con un plan ambiental. Dicha ley fue posteriormente vetada por el entonces gobernador de la provincia, Julio Cobos. Unos meses después, en junio de 2007, la Legislatura 
revisó este veto. Se presentaron diferentes proyectos de ley para frenar la minería metalífera, mientras en diferentes puntos de la provincia se realizaban manifestaciones de rechazo a esta actividad que incluían cortes de ruta. Como resultado de estas movilizaciones, que incluso mantuvieron suspendido el tránsito carretero hacia otras provincias, se sancionó la ley $\mathrm{N}^{\circ} 7722$, cuyo artículo $1^{\circ}$ establece:

A los efectos de garantizar debidamente los recursos naturales con especial énfasis en la tutela del recurso hídrico, se prohíbe en el territorio de la Provincia de Mendoza, el uso de sustancias químicas como cianuro, mercurio, ácido sulfúrico, y otras sustancias tóxicas similares en los procesos mineros metalíferos de cateo, prospección, exploración, explotación y/o industrialización de minerales metalíferos obtenidos a través de cualquier método extractivo.

Figura 2: Mapa de Mendoza, oasis y conflictos

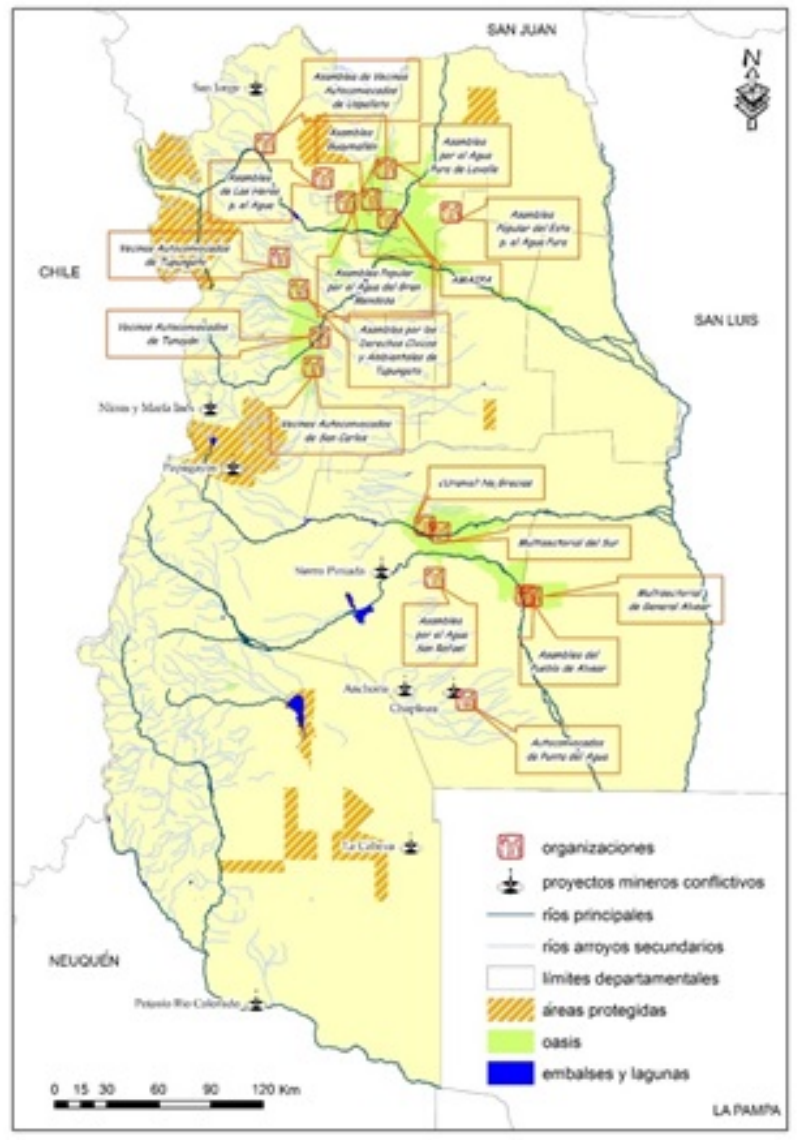

Fuente: Elaborado por Facundo Rojas y Lucrecia Wagner (2014)

Paralelamente a estos conflictos por minería metalífera, otra problemática se debatía en el departamento de San Rafael: la posible reapertura de la mina de uranio Sierra Pintada por parte de la Comisión Nacional de Energía Atómica (CNEA), organismo autárquico del Estado nacional creado en 1950. Se trata de una mina de uranio que había sido explotada a cielo abierto desde 1976 a 1997, año en que se cerró por motivos económicos. En el año 2002, en los medios de prensa locales se mencionó la posibilidad de su reapertura. La oposición a esta nueva etapa de su funcionamiento, por parte de diversos sectores de la población sanrafaelina, se fundamentó en la existencia de pasivos ambientales de la explotación anterior, que la CNEA nunca había remediado. Incluso trascendió por medios de prensa escritos el rechazo del gobierno provincial a la reapertura:

El gobierno de Iglesias [por entonces gobernador] no permitirá la reactivación del Complejo Sierra Pintada, en San Rafael, hasta que la Comisión Nacional de Energía Atómica no solucione los pasivos ambientales que tiene en ese departamento y en Malargüe. La intención de la Comisión Nacional de Energía Atómica es trasladar desde Córdoba al complejo minero 
fabril San Rafael una planta de purificación y permitir de esta forma su reactivación, teniendo en cuenta el actual valor del uranio. La resistencia del gobierno mendocino se debe, fundamentalmente, a la falta de respuesta que desde 1999 tiene la CNEA con el tratamiento de las colas de uranio de Malargüe. Pero hoy también surge claramente a la luz que hay pasivos ambientales de riesgo en la zona de San Rafael (Acevedo, 2002).

El sector agrícola fue, en San Rafael, claro opositor a la reapertura de la mina de uranio. Dos agrupaciones se organizaron allí: La Multisectorial del Sur, y ¿Uranio? No, Gracias. La primera fue integrada por diversos sectores de la población sanrafaelina: bodegueros, periodistas, organizaciones no gubernamentales (ONGs), particulares, etc., mientras que la segunda estuvo conformada por productores orgánicos de la localidad. Años después surgió la Asamblea por el Agua de San Rafael, dentro de la cual se encuentran integrantes de las organizaciones previas.

Ante la posible reapertura de Sierra Pintada, el gobierno provincial le exigió a la CNEA la remediación previa de los pasivos ambientales dejados en la explotación anterior de la mina, especialmente, las colas de uranio. El 17 de febrero de 2007 estaba planificada la audiencia pública del plan de remediación elaborado por la CNEA, a la que finalmente esta Comisión no se presentó, según trascendidos, por orden del gobierno nacional. Sin embargo, los asistentes a la audiencia decidieron tomar la palabra, aunque la CNEA no se hubiese presentado a explicar su proyecto de remediación de las colas de uranio. Así, uno de los miembros de la Multisectorial del Sur, el bodeguero Valentín Bianchi, afirmó que se encontraba en la audiencia como ciudadano y como empresario, ya que sus proyectos de traer inversores a la provincia se cayeron cuando éstos habían tomado conocimiento de la mina de uranio. Por su parte, los productores orgánicos integrantes de ¿Uranio? No, gracias argumentaron que consideraban la audiencia pública como una farsa, al ser no vinculante, y rescataron los ejemplos de Esquel y Gualeguaychú ${ }^{14}$, al afirmar que pasaba por los sanrafaelinos tomar el tema en sus manos, informarse y movilizarse.

Tanto en San Rafael, como en General Alvear -donde la Multisectorial de General Alvear está conformada, entre otros entes, por la Cámara de Comercio, Industria y Ganadería de General Alvearla base agrícola está directamente vinculada al rechazo a la actividad minera. Incluso en los inicios de la organización vecinal en San Carlos, previo a la existencia de los Vecinos Autoconvocados de San Carlosfuncionó el Frente Diamante, conformado por productores organizados en la Sociedad Rural, y liderado por el presidente de la misma. Posteriormente, el debate sobre la megaminería en Mendoza se complejizó, y otras asambleas, como la Asamblea Popular por el Agua del Gran Mendoza, o la Asamblea de Vecinos Autoconvocados de Uspallata, presentan una conformación más heterogénea, que integraba a docentes, estudiantes, comerciantes, entre otros. Incluso en las poblaciones donde algunos grupos presentan o presentaron una composición mayoritariamente de sectores agrícolas se conformaron además organizaciones más amplias, que dieron lugar a otras demandas (falta de participación en los procesos de evaluación de proyectos mineros, debates sobre el "desarrollo", etc.), como los ya mencionados Vecinos Autoconvocados de San Carlos, la Asamblea del Pueblo de Alvear y la Asamblea por el agua de San Rafael.

\section{Avance Del CONFlicto y nUevos DeBates}

El año de la sanción de la ley 7722 (2007) presentaba una situación conflictiva en relación a la minería metalífera bastante más compleja que la de los inicios del conflicto, en 2003. Como ya se destacó, en diferentes departamentos de Mendoza se habían conformado grupos que resistían a la megaminería, y éstos se habían nucleado provincialmente en AMPAP. Sumado a ello, a nivel nacional se había conformado en 2006 la Unión de Asambleas Ciudadanas (UAC), espacio que articula las diferentes luchas socioambientales existentes en el país, del cual comenzaron a participar algunos miembros de AMPAP. En marzo de 2007 la UAC se reunió en Mendoza, organizada por los grupos ya mencionados de San Rafael.

Por otra parte, en otras provincias del país ya se habían sancionado leyes restrictivas a la minería. La primera de ellas había sido la ley 5001/2003 de la provincia de Chubut, que prohibía la actividad minera metalífera a 
cielo abierto y la utilización de cianuro en los procesos de producción minera, y determinaba la zonificación del territorio provincial para la explotación de los recursos mineros. Esta ley se sancionó en el contexto del conflicto de Esquel. Posteriormente, a raíz de un nuevo conflicto por la instalación de un proyecto minero de oro en las cercanías de Ingeniero Jaccobacci, se sancionó la ley 3981/2005 de la provincia de Río Negro, que prohibía la utilización de cianuro y mercurio en el proceso de extracción, explotación o industrialización de minerales metalíferos. Esta ley fue anulada en 2011.

En 2007, el mismo año en que se sancionó la ley 7722 en Mendoza, se sancionaron leyes en La Rioja, Tucumán y La Pampa. En La Rioja, la ley 8137 prohibía la explotación minera a cielo abierto con técnicas correspondientes al proceso de lixiviación (para separar el mineral de la roca) con cianuro, mercurio o cualquier otra sustancia contaminante. Fue anulada al año siguiente de su sanción. Por su parte, la norma de la provincia de Tucumán prohíbe la actividad minera metalífera a cielo abierto y la utilización de cianuro y mercurio en los procesos de producción minera. Asimismo, la ley 2349 de la provincia de La Pampa prohíbe la utilización de cianuro, mercurio, ácido sulfúrico y toda sustancia química contaminante en los procesos mineros, y la explotación de minerales metalíferos a cielo abierto.

Tanto la ley 7722 de Mendoza, como la similar ley 9526 de Córdoba, sancionada en 2008, fueron demandadas de inconstitucionalidad por el sector minero, y defendidas por los gobiernos provinciales, que proclamaron (y lograron que las Cortes Supremas de Justicia provinciales avalaran) la constitucionalidad de estas leyes. Actualmente, hay siete provincias en Argentina que tienen legislación restrictiva a la actividad minera, entre las cuales, las últimas en sancionarse fueron las de San Luis y Tierra del Fuego.

En Mendoza, los argumentos para fundar la inconstitucionalidad por parte de las empresas mineras demandantes fueron: la inversión realizada, la violación de los derechos constitucionales de igualdad, legalidad, razonabilidad, debido proceso, propiedad y derechos adquiridos de las empresas, la discriminación (debido a que las sustancias prohibidas se utilizan en otras industrias), la prohibición irrazonable, infundada y caprichosa del uso de sustancias químicas sólo para la minería metalífera, entre otros. Las empresas entendieron la prohibición del uso de sustancias químicas como sinónimo de prohibición de la minería metalífera. Acusaron a los legisladores de intenciones "cuanto menos confusas y contradictorias", sin respaldo científico y técnico, y sin soporte intelectual ${ }^{15}$.

Por su parte, el gobierno de la provincia de Mendoza respondió la demanda, en defensa de la constitucionalidad de la ley $\mathrm{N}^{\circ}$ 7722. Nuevamente, retomó en sus argumentaciones la cultura mendocina en relación al agua y a su perfil productivo:

Los mendocinos somos por historia poseedores de una cultura que ha sabido vencer las adversidades del clima. La aridez natural del mismo exigió a sus hombres y mujeres una especial actitud para mejorar las condiciones ambientales necesarias para el desenvolvimiento de sus actividades. Así, aprendimos a organizar y administrar el escaso recurso hídrico, hasta conformar los oasis irrigados en los que vivimos (Asesoría de Gobierno, 2007, p. 10).

En cuanto al planteo de desigualdad y discriminación, el gobierno provincial respondió destacando la magnitud de los impactos ambientales de la actividad minera, a la que denominó como actividad extractiva:

Y claro está que la actividad minera extractiva no es una actividad equiparable en su desarrollo y consecuencias ambientales a cualquier otra actividad. Basta atender al respecto que el mismo Código de Minería estipula aspectos básicos para dicha actividad en materia de prevención y reparación de daños ambientales, los que son regulados para la minería y no para las restantes actividades que enumera la actora (hidrocarburos, agricultura, vitivinicultura, laboratorios, etc.) (Asesoría de Gobierno, 2007, p. 15).

En este tema, el gobierno mendocino fue más allá, al resaltar que las características diferentes del impacto ambiental de la actividad minera llevaron a que ésta tenga una legislación específica, en referencia a la ley de impacto ambiental introducida en el año 1995 al Código de Minería. Además, también hizo referencia a los beneficios impositivos para la actividad minera, otorgados por leyes nacionales, cuestionando que las 
empresas mineras solicitan igualdad ante la evaluación de los impactos ambientales, pero no consideran los beneficios impositivos que no comparten con otras actividades:

\begin{abstract}
No se observa en la demanda cuál es la situación igualitaria que entiende la actora que le corresponde frente a otras actividades que en la realidad no presentan idénticas características. La igualdad que ahora sostiene no es la que ha fundado la existencia de un régimen de impacto y daño ambiental específico (Ley 24.585). Tampoco lo es la que otorga a la actividad minera claras diferencias impositivas y aduaneras con las demás actividades. Indudablemente, la actora no se siente igual a la hora de recibir beneficios que no son otorgados a las restantes actividades, pero sí a la hora de asumir el cese de los riesgos y externalidades de su actividad (Asesoría de Gobierno, 2007, pp. 16-17).
\end{abstract}

Posteriormente, la defensa avanza sobre el uso diferenciado de las sustancias prohibidas, y en el riesgo que implica la actividad minera para la provincia. Reconoce que la minería metalífera ha sido identificada como de alto impacto contaminante, y destaca que en Mendoza esta actividad no está prohibida, sino limitada, ya que lo que se prohíbe es el uso de ciertas sustancias en los procesos mineros. Esto se vio confirmado en 2009 por la llegada del proyecto minero San Jorge, que aunque se trataba de minería metalífera avanzó en su intento de explotación de cobre en Uspallata, porque no planteaba el uso de ninguna de las sustancias explícitamente prohibidas en la ley 7722. El gobierno provincial destacó, una vez más, el riesgo que la actividad minera implicaba para la sostenibilidad de Mendoza, lo cual justificaba la existencia de normas ambientales más exigentes $^{16}$.

Como puede observarse, los planteos del gobierno provincial en su defensa de la constitucionalidad de la ley avanzaron en nuevos debates sobre la actividad: sus beneficios impositivos, la diferencia de la magnitud del impacto ambiental respecto a otras actividades y el riesgo que implicaría para la provincia. Un tema que agregó la defensa de la ley 7722, en comparación con la defensa de la constitucionalidad de la ley atacada años antes (la 7422), fue la responsabilización del sector minero por la reacción adversa de la población ante la llegada de la actividad, por no haber informado correctamente sobre la misma.

Pero lo más llamativo a nuestro entender, radica en la circunstancia de haber sido el propio sector minero constituido por organismos gubernamentales y de la sociedad civil (Asociaciones, centros y consejos de geólogos e ingenieros en minas), el responsable de la situación de intolerancia e incomprensión que evidencia la sociedad respecto de los fenómenos sociales y económicos inherentes a la industria minera (Asesoría de Gobierno, 2007, p. 25).

Evidentemente, este nuevo argumento estuvo fundamentado en la escalada del conflicto minero, de 2005 a 2007, año en el que se realizaron las manifestaciones sociales que, en junio de 2007, mantuvieron algunos días aislada a la provincia debido a los cortes de ruta en diferentes puntos de la misma, hechos que impulsaron la sanción de la ley 7722 para destrabar el conflicto.

\title{
5. Diálogos con otros ANÁlisis DEL TEMA
}

El análisis de la conflictividad socioambiental desatada en Mendoza por la llegada de proyectos de minería metalífera requiere debatir si se trata de una disputa meramente ambiental, o si encubre conflictos de intereses entre actividades y modelos económicos aparentemente contrapuestos. En este sentido, avanza Mauricio Pinto (2012) cuando expone que gran parte del discurso crítico de la minería no pasa por la tutela ecosistémica en sí misma, sino que refiere a la distribución de los recursos naturales en juego entre los diversos sectores económicos (por ejemplo, agricultura vs. minería). El autor destaca que este aspecto de la confrontación, aunque vestido en un traje ambientalista, en cierta forma se aleja de las pretensiones estrictamente ambientales para ingresar en otra faceta del paradigma de desarrollo de cada sociedad.

Por su parte, Liber Martín plantea que "La disputa puede ser leída de manera más real, ya no tanto en defensa de un interés ambiental abstracto y retórico, sino entre dos modelos de desarrollo económico con diversas implicancias ambientales" (Martín, 2008, p. 4). Ambos autores, desde un análisis jurídico, destacan 
discusiones que han estado presentes en el devenir del conflicto. Entre ellas, la competencia por el uso del agua:

(...) en gran medida, se trató de una disputa por el agua, entre los sectores agrícola productivos tradicionales con fuerte arraigo en la cultura e idiosincrasia de Mendoza que -además de ver amenazada la calidad de las aguas, vieron fundamentalmente amenazados los volúmenes de agua- y el uso minero, que requería de ingentes cantidades del mismo líquido, siempre escaso en la provincia. El modelo de desarrollo agrícola tradicional encontró así en la causa ambiental, un discurso con fuerte aceptación popular y en los ambientalistas, un aliado estratégico en la disputa por el recurso, en cuanto importaba conservar el status quo por sobre la novedosa pero demonizada actividad minera (Martín, 2008, p. 4).

Liber Martín concluye que, desde este punto de vista, la ley (7722) puede ser considerada como el resultado legítimo de esa puja de intereses y el triunfo del modelo tradicional de desarrollo de la provincia de Mendoza -y de los nuevos actores ambientales- por sobre el nuevo modelo de desarrollo minero que iba cobrando forma. Por su parte, Mauricio Pinto agrega su apreciación sobre la garantía jurídica del orden de prioridades en el uso del agua:

La tensión entre los tradicionales usuarios del suelo y del agua, así como la disponibilidad de recursos humanos, y las nuevas necesidades que anuncia la minería es un elemento presente en el conflicto que debe considerarse de manera directa, incluyendo en todo proceso de diálogo a las instituciones que aglutinan a los respectivos y legítimos interesados. En lo que hace a la competencia que surge de la demanda de agua, y más allá de que como observamos supra quizás exista un sobredimensionamiento de este tópico en la discusión que debería despejarse mediante un análisis técnico hasta ahora ausente, debe recordarse que la distribución de tal recurso entre las distintas actividades sociales no se encuentra librada a la mera competencia entre usuarios, ni a la arbitrariedad administrativa en la asignación. Ello es propio del rol planificador estatal, donde existen criterios estaduales fijados normativamente que priorizan actividades con respecto a otras. Ese orden de prioridades impuesto por ley excluye que la posibilidad -al menos jurídicamente- de que un usuario agrícola pueda ser privado de su derecho de aguas en beneficio de un nuevo emprendimiento minero (Pinto, 2012).

Paradójicamente, a pesar de esta garantía jurídica, los conflictos por la potencial instalación de la actividad minera en Mendoza han perdurado por más de diez años. Es evidente que, si bien Mendoza es una provincia donde la institucionalidad ha respondido al conflicto con la sanción de legislación restrictiva a la actividad, a pesar de ello persiste la desconfianza en el cumplimiento de las leyes y, sobre todo, en la capacidad de control ambiental hacia la megaminería por parte del Estado. En Mendoza, la juridificación y judicialización del conflicto (Merlinsky, 2013) ${ }^{17}$ y su materialización en normativas acompaña, al mismo tiempo que nutre, a la genealogía de las movilizaciones. Es decir que, ante las manifestaciones sociales, el Estado ha recurrido a retóricas basadas en el imaginario ambiental históricamente construido -que son compartidas por las comunidades que se movilizan-, pero éstas también cuestionan los instrumentos con los que el Estado responde desde su institucionalidad, lo que desborda su capacidad de respuesta.

En los inicios de esta conflictividad, algunos sectores del Estado provincial intentaron conciliar las actividades tradicionales de la provincia con la nueva actividad "alternativa" (la megaminería), desde un discurso basado en el desarrollo sustentable y el control ambiental. Quizás el primer intento en este sentido, que fracasó totalmente, fueron unas jornadas organizadas por el Ministerio de Economía, en junio de 2005, llamadas Minería sustentable en Mendoza, un desafío para el siglo XXI. Este encuentro se realizó en San Carlos, con el objetivo de plantear la compatibilidad de la minería con el medio ambiente. En sus declaraciones a los medios, la entonces ministra de Economía de la provincia definía la posición del gobierno de compatibilizar la actividad minera con la protección del medio ambiente: "la Provincia quiere promover el recurso, pero generando un concepto minero nuevo que tenga en cuenta el asiento de otras actividades (como agricultura y turismo)" ${ }^{18}$. Esta posibilidad de compatibilizar las actividades tradicionales con la megaminería no tuvo éxito y, ante ello, otros sectores del Estado provincial avanzaron con la respuesta al conflicto por vía normativa, la cual aumentó los requerimientos para la aprobación de la actividad minera metalífera, y terminó colaborando con su paralización. 
En cuanto a la diversidad de los actores intervinientes en el conflicto, Liber Martín (2008) destaca dos aspectos que también son fundamentales para el análisis aquí realizado: en primer lugar, y como ya fue destacado previamente, entre los sectores que motorizan las presiones (para frenar la megaminería) no se encuentran solamente ambientalistas, sino también empresarios, productores y comerciantes, vinculados fundamentalmente al sector agrícola, agroindustrial y ganadero. En segundo lugar, la actividad agroindustrial no resulta en ninguna medida inocua, sino que produce diversas externalidades negativas en relación al agua y a los hábitats naturales.

Estos sectores agroindustriales y ganaderos son una parte de esta resistencia a la megaminería, que incluye también pequeños productores, campesinos sin tierra, comunidades indígenas y otros grupos de la sociedad mendocina que complejizaron las críticas, vinculando la megaminería a un modelo de desarrollo extractivista con serias implicancias ambientales y sociales. Lo que resulta central en esta resistencia es que "el agua se ha constituido como centro estructurador de las movilizaciones en rechazo a la megaminería, encontrando legitimidad en la histórica "cultura del agua" pero presentando también la potencialidad de encauzar y articular otras tramas y dinámicas sociales” (Martín y Wagner, 2013, p. 316).

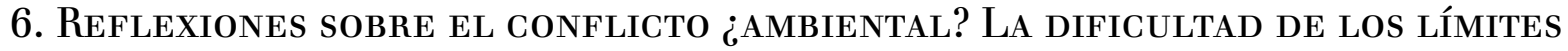

Como se destacó a lo largo del texto, la reflexión que dio lugar a este trabajo surge de una investigación sobre la conflictividad socioambiental en Mendoza debido a la megaminería, la conformación de colectivos sociales que resistieron a esta actividad (e impidieron su instalación), y la interacción de estos procesos con la evaluación gubernamental de los proyectos mineros a gran escala. A partir del trabajo sobre fuentes gubernamentales, empresariales y de organizaciones sociales, sumado a la realización de entrevistas a diferentes actores del conflicto, se pudo comprobar que la argumentación sobre la cual se basaba el cuestionamiento a la megaminería, tanto por parte del gobierno como también de colectivos sociales que resistían a esta actividad en diferentes lugares del territorio mendocino, se basaba en una historia provincial agrícola que postulaba el uso del agua para riego y el riesgo que la megaminería podía implicar para este modelo.

A ello, se suma la preservación de áreas naturales protegidas que actúan como reservas hídricas, defensa que se ha ido acentuando y que en los últimos años se manifiesta en la postulación de áreas protegidas y el potencial turístico que éstas implican- como alternativas a los proyectos extractivos. Así, en Mendoza asistimos a un proceso de conflictividad en el que, dentro de las demandas emergentes, aquellas que pueden ser consideradas "ambientales" han ganado escena en las últimas décadas.

Concluimos que, en esta ambientalización de la conflictividad en torno a los modelos de desarrollo, el imaginario ambiental mendocino y la denominada cultura del oasis han sido ingredientes centrales de los conflictos socioambientales generados por megaproyectos mineros en Mendoza. Por ello, el análisis de la compatibilidad -o no- del modelo agrícola con el de la minería metalífera, si realmente procura comprehender el conflicto, debe incorporar estos elementos culturales e identitarios en los que se ha basado, en parte, la resistencia a la megaminería.

Por otra parte, el cuestionamiento a un modelo extractivo ha implicado el acercamiento de algunas de las asambleas socioambientales con medianos y pequeños productores, movimientos campesinos, espacios de agroecología y otras formas alternativas de producción y desarrollo, generalmente invisibilizadas en un perfil mendocino en el que el modelo vitivinícola aparece como hegemónico. Sumada a ello, la participación en diferentes espacios provinciales de actores políticos dispuestos a dar debate, ampliar los espacios participativos y consolidar espacios de educación formal y no formal en cuestiones ambientales es otra consecuencia del conflicto por megaminería que ha trascendido, con el correr de los años, la dicotomía entre agricultura y megaminería. 


\section{Agradecimientos}

Al CONICET, por financiar esta investigación. Al Grupo de Historia Ambiental del IANIGLA-CCT Mendoza, por los aportes recibidos, especialmente a Facundo Rojas. A las personas y organizaciones entrevistadas, y a las dependencias gubernamentales que me permitieron el acceso a la documentación y expedientes aquí analizados.

\section{REFERENCIAS}

Abraham, E. M., y Rodríguez Salas, A. (2003). Política ambiental en la provincia de Mendoza: Contenidos y Alcances de la Programación Ambiental. APORTES, 12, 1-16. Recuperado de: http://www.asociacionag.org.ar/pdfapo rtes/12/a12_03.pdf

Acevedo, F. (17, 12, 2002). El Gobierno frena la actividad de la CNEA por el daño ecológico. Diario Los Andes. Recuperado de http://archivo.losandes.com.ar/notas/2002/12/17/sociedad-57662.asp

Acselrad, H. (2010). Ambientalização das lutas sociais -o caso do movimiento por justiça ambiental. Estudos Avançados, 24(68), janeiro-abril, 103-119.

Alimonda, Héctor (coord.) (2011). La Naturaleza colonizada: Ecología politica y minería en América Latina. Buenos Aires: CLACSO,.

Antonelli, M. (2014). Megaminería transnacional e invención del mundo cantera. Nueva Sociedad, 252, julio-agosto. Recuperado de http://nuso.org/

Asesoría de Gobierno (2005). Contesta Acción de Inconstitucionalidad: Minera Angloamerican Argentina S.A. c/ Gobierno provincia de Mendoza s/acción de inconstitucionalidad. Mendoza: Gobierno de la provincia de Mendoza.

Asesoría de Gobierno (2007). Contesta acción de Inconstitucionalidad en contra la Ley Provincial $N^{\circ} 7.722$. Mendoza: Gobierno de la provincia de Mendoza.

Bottaro, L., y Sola Álvarez, M. (coords.) (2018). Agua y Megaproyectos mineros en América Latina. Los Polvorines: Universidad Nacional de General Sarmiento-WATERLAT/GOBACIT.

Bueno, M. del P. (2014). La política minera en la argentina y el modelo extractivista. Foro Internacional, LIV(1), El Colegio de México, enero-marzo, 106-130.

Carman, M. (2011). Las trampas de la naturaleza. Medio ambiente y segregación en Buenos Aires. Buenos Aires: Fondo de Cultura Económica.

Claps, L. M., y D. S. Colao (2005). Comunicación, recursos naturales y comunidad en el caso Esquel (Tesis de Licenciatura). Facultad de Ciencias Sociales, Universidad de Buenos Aires (UBA), Buenos Aires.

Deonandan, K. \& Dougherty, M. (eds.) (2016). Mining in Latin America: Critical Approaches to the New Extraction. Oxon-New York: Routledge.

Delgado Ramos, G. C. (coord.) (2010). Ecología Politica de la minería en América Latina. México: UNAM.

Dirección de Estadísticas e Investigaciones Económicas-DEIE (2005). Producto Bruto Geográfico Sector Agropecuario. Mendoza: Ministerio de Economía, Infraestructura y Energía, Gobierno de Mendoza.

Escolar, D., Martín, F., Rojas, F., Saldi, L. y Wagner, L. (2012). Imaginario ambiental mendocino. Sus efectos en las políticas estatales y la producción científica. En A. Salomón y A. Zarrilli (Comp.), Historia, politica y gestión ambiental. Perspectivas y debates (pp. 77-98). Buenos Aires: Imago Mundi.

Galafassi, G. (2013). Dossier: Modos de acumulación, recursos naturales y dominio colonial en América Latina. Un intento de mirada crítica sobre la "reinvención" del modelo extractivo. Theomai, 25. Recuperado de http://revi sta-theomai.unq.edu.ar/NUMERO\%2025/contenido_25.htm

Giraud, M., y Wagner, L. (2017). Dossier “Minería y otros extractivismos”. RevIISE, 10(10), 65-312. Recuperado de: http://www.ojs.unsj.edu.ar/index.php/reviise/issue/view/17/showToc

Giraud, M., y Wagner, L. (2017). Dossier "Narrativas, Relatos y Experiencias de Resistencias”. RevIISE, 10(10), 315-330. Recuperado de: http://www.ojs.unsj.edu.ar/index.php/reviise/issue/view/17/showToc 
Göbel, B. y Ulloa, A (eds.) (2014). Extractivismo minero en Colombia y América Latina. Bogotá-Berlín: Universidad Nacional de Colombia-Ibero-Amerikanisches Institut.

Godfrid, J. (2016). El discurso pro-minero en Argentina. Un estudio de caso a partir de la empresa minera La Alumbrera. RBLA, 16(2), 257-276.

Hadad, G. (2013). Dossier: Ecología Política y Ciencias Sociales: las disputas socioambientales. Entramados y Perspectivas, 3, 7-12. Recuperado de: http://publicaciones.sociales.uba.ar/index.php/entramadosyperspectivas/ issue/view/16/showToc

Leite Lopes, J. S. (2006). Sobre procesos de “ambientalização" dos conflitos e sobre dilemas da participação. Horizontes Antropológicos, 12(25), jan./jun, 31-64.

Machado, H., Svampa, M., Viale, E., Giraud, M., Wagner, L., Antonelli, M., Giarracca, N. y Teubal, M. (2011) 15 Mitos y Realidades de la minería transnacional en la Argentina. Buenos Aires: Editorial El Colectivo y Ediciones Herramienta.

Machado Araoz, H. (2012). Naturaleza mineral. Una ecología política del colonialismo minero (Tesis de Doctorado en Ciencias Humanas). Universidad Nacional de Catamarca, Catamarca, Argentina.

Martín, F., y Wagner, L. (2013). Agua o minería. Determinaciones y movilizaciones en la construcción pública del conflicto ambiental en Mendoza. En G. Merlinsky (Comp.), Cartografias del Conflicto Ambiental en Argentina (pp. 287-320). Buenos Aires: CLACSO-CICCUS.

Martín, L. (2008). La regulación de la utilización de sustancias químicas en la actividad minera metalífera en el marco del desarrollo sostenible en la provincia de Mendoza, Argentina. Rivista di diritto pubblico italiano, comunitario e comparato, (2) Sommario del 23/01/2008, 1-10. Recuperado de http://www.federalismi.it/nv14/articolo-do cumento.cfm?artid $=9085$

Merlinsky, G. (comp.) (2013). Cartografías del Conflicto Ambiental en Argentina. Buenos Aires: CLACSO-CICCUS.

Montaña, E., Torres, L., Abraham, E., Torres, E., y Pastor, G. (2005). Los espacios invisibles. Subordinación, marginalidad y exclusión de los territorios no irrigados en las tierras secas de Mendoza, Argentina. Región y sociedad, XVII(32), 3-32. Recuperado de http://www.redalyc.org/articulo.oa?id=10203201

Pinto, M. (2012). Doctrina del día: tribulaciones jurídicas sobre el conflicto minero-ambiental en Mendoza. La Ley Gran Cuyo, 239, abril. Recuperado de http://thomsonreuterslatam.com/2012/06/11/2470/

Richard-Jorba, R., Pérez Romagnoli, E., Barrio, P., y Sanjurjo, I. (2006). La región vitivinicola argentina: transformaciones del territorio, la economía y la sociedad, 1870-1914. Buenos Aires: Universidad Nacional de Quilmes.

Rodríguez Pardo, J. (2006). En la Patagonia NO. Crónica de la epopeya antinuclear de Gastre. El Bolsón: Proyecto Lemu-Grupos de Amigos del Libro.

Rodríguez Pardo, J. (2009). Vienen por el oro, vienen por todo. Las invasiones mineras 500 años después. Buenos Aires: Ediciones Ciccus.

Schiaffini, H. (2003). "El agua vale más que el oro". La constitución de fuerzas sociales en torno al conflicto minero en Esquel. 2002-2003 (Tesis de Licenciatura). Departamento de Ciencias Antropológicas, UBA, Buenos Aires, Argentina.

Sola Alvarez, M. (2012). Conflictos socioambientales en torno a la megaminería metalifera. El caso de Famatina, La Rioja, Argentina. Maestría en Planificación Urbana y Regional. Buenos Aires: UBA.

Svampa, M. y Antonelli, M. (eds.) (2009). Minería transnacional, narrativas del desarrollo y resistencias sociales. Buenos Aires: Editorial Biblos.

Wagner, L. (2010). Problemas Ambientales y Conflicto Social en Argentina: Movimientos socioambientales en Mendoza. La defensa del agua y el rechazo a la megaminería en los inicios del Siglo XXI (Tesis de Doctorado en Ciencias Sociales y Humanas), Universidad Nacional de Quilmes, Buenos Aires, Argentina. Recuperada de https://rida a.unq.edu.ar/handle/20.500.11807/192

Wagner, L. (2012). Uspallata: ecos sociales de la megaminería en un valle andino. CLAROSCURO, 11, 191-215. 
Wagner, L. (2014). Conflictos socioambientales: la megaminería en Mendoza, 1884-2011. Buenos Aires: Editorial de la Universidad Nacional de Quilmes.

Walter, M. (2014). Political Ecology of Mining Conflicts in Latin America. An Analysis of environmental movements and struggles over scales. PHD Programme in Environmental Sciences. Barcelona: Universidad Autónoma de Barcelona.

\section{Notas}

1 Según el relevamiento, aún en proceso, de conflictos ambientales a nivel mundial, sistematizado en el Atlas de Justicia Ambiental, de 2551 conflictos relevados hasta el momento, 540 corresponden a conflictos por minería, de los cuales 228 se localizan en Sudamérica. Argentina posee hasta el momento 33 conflictos registrados. Fuente: Atlas de Justicia Ambiental, http://ejatlas.org/

2 Fuente: noticia titulada "San Rafael: la Multisectorial del Sur interpuso amparo ambiental por glaciares”, publicada en La Quinta Pata, recuperada de: http://la5tapata.net/san-rafael-la-multisectorial-del-sur-interpuso-amparo-ambientalpor-glaciares/

3 Asamblea Popular por el Agua del Gran Mendoza, Vecinos Autoconvocados de San Carlos, Multisectorial de General Alvear y Asamblea del Pueblo de Alvear, y Asamblea de Vecinos Autoconvocados de Uspallata. El año 2011 corresponde al rechazo en la legislatura provincial del proyecto San Jorge, primer proyecto metalífero que presentó su Informe de Impacto Ambiental de la etapa de explotación, en la localidad de Uspallata.

4 El artículo 24 de la ley 6.045 (Régimen de áreas naturales provinciales) prohíbe la exploración y explotación minera en las áreas restringidas.

5 Si se tienen en cuenta las estadísticas provinciales, para el año 2005 las principales actividades dentro del sector agropecuario fueron: la viticultura, con una participación relativa de 47,95\%, la fruticultura 17,72 \% y hortalizas y legumbres con 10,85\%. El subsector agrícola provincial puede dividirse en cuatro mercados básicos: Vitícola; Frutícola (excluyendo la uva), los productos más importantes que componen este mercado son el durazno, la ciruela, la manzana, el damasco y la pera; Hortícola, actividad en la que se destaca el ajo, la cebolla, el tomate, la papa, el pimiento, la zanahoria y el zapallo coreano; y Olivícola. Del total de tierras implantadas en la provincia, la vid ocupa, en promedio, el $64 \%$. Le siguen en importancia las hortalizas con un promedio de $17 \%$ de la superficie total implantada, los frutales (especialmente durazneros, ciruelos, damascos, manzanos y perales) con un promedio del $12 \%$. El olivo ocupa aproximadamente el $8 \%$ de la superficie cultivada (DEIE, 2005).

6 Mendoza podría ser considerado parte del denominado "efecto post Esquel". Esquel fue la primera localidad argentina, en la provincia patagónica de Chubut, en rechazar un proyecto minero de oro, en el año 2002, llegando en 2003 a la realización de un plebiscito por el cual la actividad minera fue rechazada por el $82 \%$ de los votos.

7 Según el Censo 2001, San Carlos tenía en aquel entonces 28.341 habitantes (Instituto Nacional de Estadísticas y CensosINDEC, 2001).

8 Fuente: Noticia titulada: "San Carlos, el pueblo que se rebeló contra la minería”, Diario Los Andes, 11 de septiembre de 2005.

9 El entrevistado se refiere al método de minería metalífera a cielo abierto con uso de sustancias químicas, como cianuro o ácido sulfúrico, entre otros, en los procesos de lixiviación (que separan el mineral de la roca).

10 Javier Rodríguez Pardo escribió dos libros sobre sus experiencias acompañando las diferentes luchas socioambientales en Argentina, En la Patagonia NO. Crónica de la epopeya antinuclear de Gastre (2006) y Vienen por el oro, vienen por todo. Las invasiones mineras 500 años después (2009).

11 Entrevistas realizadas durante el trabajo de campo correspondiente a la tesis doctoral Problemas Ambientales y Conflicto Social en Argentina: Movimientos socioambientales en Mendoza. La defensa del agua y el rechazo a la megaminería en los inicios del Siglo XXI (Wagner, 2010). Los entrevistados fueron seleccionados teniendo en cuenta su trayectoria en los movimientos, la vivencia de momentos clave del conflicto y buscando dar cuenta de la diversidad entre los mismos (considerar a personas de diferentes edades, sexo, extracción política -si la tienen-, ocupación, entre otras características). Posteriormente, se llevaron a cabo nuevas entrevistas que fueron publicadas en Wagner (2014).

12 En junio de 2007 el conflicto en torno a la megaminería alcanzó uno de sus picos más álgidos. Se realizaron cortes en diferentes puntos de la provincia, durante casi 20 días, y llegó en algunos momentos a cortes totales (impedimento del tránsito carretero). Esta situación agilizó la decisión de la Legislatura provincial de sancionar la ley 7722, que prohíbe el uso de ciertas sustancias en la actividad minera.

13 La Ley N 7.627 , también conocida como "Ley Difonso", fue sancionada por la Legislatura de la provincia de Mendoza el 13 de diciembre de 2006. En su primer artículo, esta ley expresaba: "Suspéndase el otorgamiento de derechos mineros, de cateos, exploraciones o explotaciones metalíferas a cielo abierto, hasta tanto la Provincia de Mendoza tenga aprobado 
el Plan Ambiental de la Ley 5961". La Ley fue posteriormente vetada por el ejecutivo provincial. Por su parte, Jorge Difonso, impulsor de esta ley, en las elecciones del año 2007 fue elegido intendente del departamento de San Carlos. Al cierre de este trabajo, Difonso va por su tercer mandato como intendente de San Carlos.

14 En referencia a las movilizaciones que se realizaron en Gualeguaychú y otras ciudades de la provincia de Entre Ríos, para rechazar la instalación de fábricas de pasta de celulosa en Uruguay, sobre el río Uruguay que comparten Argentina y Uruguay.

15 Fuente: Poder Judicial, Causa "Minera del Oeste S. R. t. y otras contra Gobierno de la Provincia sobre acción de inconstitucionalidad", Juicio: 20/07/2007, Mendoza.

16 (...) no todos los procesos importan idéntica aplicación de las sustancias en cuestión. La minería metalífera es señalada por la doctrina especializada como de alto impacto contaminante, y con ello es razonable impedir que utilice ciertas sustancias que en otros procesos productivos no se meritúan de igual riesgo o peligro (...) La actividad minera no es objeto de prohibición, sino el uso de ciertas sustancias por la misma. Las sustancias prohibidas no son las únicas que pueden emplearse en la actividad minera metalífera (Asesoría de Gobierno, 2007, pp. 17-18). El riesgo que implica la actividad minera para la sostenibilidad de Mendoza justifica normas ambientales específicas con exigencias diferenciales a las de otras actividades productivas (Asesoría de Gobierno, 2007, p. 22).

17 Juridificación se refiere al proceso social mediante el cual los conflictos ambientales son resignificados cuando son llevados a la esfera del derecho, analizando la forma en que los actores utilizan el repertorio jurídico a su disposición. Cuando el conflicto es llevado a la justicia, implicando la participación de tribunales, se produce la judicialización de los mismos (Merlinsky, 2013).

18 Fuente: noticia titulada "El riesgo ambiental se instala en la agenda de los municipios mineros", del Diario Los Andes, $1 / 06 / 2005$. 